\title{
Density-matrix-functional calculations for matter in strong magnetic fields: Ground states of heavy atoms
}

\author{
Johnsen, Kristinn; Yngvason, Jakob
}

Published in:

Physical Review A

Link to article, DOI:

10.1103/PhysRevA.54.1936

Publication date:

1996

Document Version

Publisher's PDF, also known as Version of record

Link back to DTU Orbit

Citation (APA):

Johnsen, K., \& Yngvason, J. (1996). Density-matrix-functional calculations for matter in strong magnetic fields: Ground states of heavy atoms. Physical Review A, 54(3), 1936-1946. https://doi.org/10.1103/PhysRevA.54.1936

\section{General rights}

Copyright and moral rights for the publications made accessible in the public portal are retained by the authors and/or other copyright owners and it is a condition of accessing publications that users recognise and abide by the legal requirements associated with these rights.

- Users may download and print one copy of any publication from the public portal for the purpose of private study or research.

- You may not further distribute the material or use it for any profit-making activity or commercial gain

- You may freely distribute the URL identifying the publication in the public portal

If you believe that this document breaches copyright please contact us providing details, and we will remove access to the work immediately and investigate your claim 


\title{
Density-matrix-functional calculations for matter in strong magnetic fields: Ground states of heavy atoms
}

\author{
Kristinn Johnsen ${ }^{1,2,3}$ and Jakob Yngvason ${ }^{2,3}$ \\ ${ }^{1}$ Mikroelectronik Centret, Technical University of Denmark, Bygning 345 D, DK 2800 Lyngby, Denmark \\ ${ }^{2}$ Science Institute, University of Iceland, Dunhaga 3, IS 107 Reykjavik, Iceland \\ ${ }^{3}$ NORDITA, Blegdamsvej 17, DK 2100 København D, Denmark
}

(Received 6 March 1996)

\begin{abstract}
We report on a numerical study of the density matrix functional introduced by Lieb, Solovej, and Yngvason for the investigation of heavy atoms in high magnetic fields. This functional describes exactly the quantum mechanical ground state of atoms and ions in the limit when the nuclear charge $Z$ and the electron number $N$ tend to infinity with $N / Z$ fixed, and the magnetic field $B$ tends to infinity in such a way that $B / Z^{4 / 3} \rightarrow \infty$. We have calculated electronic density profiles and ground-state energies for values of the parameters that prevail on neutron star surfaces and compared them with results obtained by other methods. For iron at $B=10^{12} \mathrm{G}$ the ground-state energy differs by less than $2 \%$ from the Hartree-Fock value. We have also studied the maximal negative ionization of heavy atoms in this model at various field strengths. In contrast to Thomas-Fermi type theories atoms can bind excess negative charge in the density matrix model. For iron at $B=10^{12} \mathrm{G}$ the maximal excess charge in this model corresponds to about one electron. [S1050-2947(96)01809-4]
\end{abstract}

PACS number(s): 31.15.-p, 03.65.-w, 32.10.-f, 97.60.Jd

\section{INTRODUCTION}

The properties of matter in magnetic fields of the extreme strength of $10^{12} \mathrm{G}$ and higher have been the subject of numerous investigations since the early 1970s, a major impetus being the discovery of pulsars in 1968 and the resulting interest in magnetized neutron stars. We refer to [1-5] for general reviews on this subject and lists of references. The standard Hamiltonian of atomic physics,

$$
\begin{aligned}
H_{N, B, Z}= & \sum_{i=1}^{N}\left(\left\{\left[\mathbf{p}^{(i)}+\mathbf{A}\left(\mathbf{r}^{(i)}\right)\right] \cdot \boldsymbol{\sigma}^{(i)}\right\}^{2}-Z\left|\mathbf{r}^{(i)}\right|^{-1}\right) \\
& +\sum_{1 \leqslant i<j \leqslant N}\left|\mathbf{r}^{(i)}-\mathbf{r}^{(j)}\right|^{-1},
\end{aligned}
$$

is usually taken as a starting point for the study of atoms in the atmosphere and outermost crust of neutron stars. Here $N$ is the number of electrons that move in the Coulomb field of a nucleus, localized at the origin with charge $Z e$, and in a homogeneous magnetic field $\mathbf{B}=(0,0, B)$ with vector potential $\mathbf{A}(\mathbf{r})=(1 / 2)(-y B, x B, 0)$. The Hamiltonian (1) operates on antisymmetric $N$-particle wave functions $\Psi$ of the space and spin variables, and $\boldsymbol{\sigma}=\left(\sigma_{1}, \sigma_{2}, \sigma_{3}\right)$ is the vector of Pauli matrices. Units are chosen such that $\hbar=e=2 m_{e}=1$, $c=1 / \alpha \approx 137$; the energy unit is then four times the Rydberg energy, i.e., $54.4 \mathrm{eV}$. Besides the atomic Hamiltonian (1) it is, of course, important to study the Hamiltonian for molecules and matter in bulk, but the present paper is only concerned with (1), more specifically with its ground-state energy

$$
E^{\mathrm{Q}}(N, B, Z)=\inf _{(\Psi, \Psi)=1}\left(\Psi, H_{N, B, Z} \Psi\right),
$$

and the ground-state electron density is

$$
\begin{aligned}
\rho_{N, B, Z}^{Q}(\mathbf{r})= & N \sum_{s^{(i)}= \pm 1 / 2} \\
& \times \int\left|\Psi_{0}\left(\mathbf{r}, \mathbf{r}^{(2)}, \ldots, \mathbf{r}^{(N)} ; s^{(1)}, \ldots, s^{(N)}\right)\right|^{2} \\
& \times d^{3} \mathbf{r}^{(2)} \ldots d^{3} \mathbf{r}^{(N)}
\end{aligned}
$$

where $\Psi_{0}$ is a ground-state wave function.

Previous works on matter in strong magnetic fields can roughly be divided into two classes. On the one hand, the focus has been on light atoms, in particular hydrogen with $Z=1$, on the other hand, focus has also been on heavy atoms with high $Z$. The present contribution falls into the second class. Here $Z=26$ plays a special role because iron is believed to be the most abundant element in the surface layer of a neutron star $[1,2]$. For such heavy atoms it is reasonable to expect that important aspects can be extracted from an asymptotic analysis in $Z$, and since $10^{12} \mathrm{G}$ is large even compared with the natural atomic unit $B_{0}=m^{2} e^{3} c / \hbar^{3}$ $=2.35 \times 10^{9} \mathrm{G}$, an asymptotic analysis in $B$ is equally called for. ${ }^{1}$

The asymptotic behavior of the energy (2) and density (3) as $N, Z \rightarrow \infty$, where $N / Z$ is fixed and the magnetic field $B$ is allowed to vary with $Z$ as well, has recently been rigorously studied by Lieb, Solovej, and Yngvason [6,4,5]. In these papers it was proved that the ground-state properties of (1) can in this limit be evaluated exactly by five nonlinear functionals corresponding to different physics at different scales of the magnetic field $B$ as measured by powers of $Z$. These five parameter regions are characterized as follows: Region 1 , $B \ll Z^{4 / 3}$; region $2, B \sim Z^{4 / 3}$; region $3, Z^{4 / 3} \ll B \ll Z^{3}$; region 4 ,

\footnotetext{
${ }^{1}$ With our choice of units $2 m_{e}=1$ and the magnetic field is actually measured in units of $4 B_{0}=9.40 \times 10^{9} \mathrm{G}$.
} 
$B \sim Z^{3}$; region $5, B \gg Z^{3}$. Here $B \ll Z^{p}, B \gg Z^{p}$, and $B \sim Z^{p}$ mean, respectively, that the ratio $B / Z^{p}$ tends to $0, \infty$, or a constant $\neq 0$ as $Z \rightarrow \infty$.

The asymptotic theories corresponding to regions $1-3$ are semiclassical theories of Thomas-Fermi type that have been extensively applied to neutron stars in the past, see, e.g., [7-13]. Salient features of atoms in region 5 were captured by a different density functional theory already in the papers $[14,15]$. However, the conditions on the surface of a typical neutron star correspond rather to region 4, and this asymptotic region is also the most interesting one from the mathematical point of view. In fact, in [4] it is shown that it can be described by a functional of a novel type, where the variable is not a density, but a function with values in density matrices. Moreover, this theory covers regions 3 and 5 as limiting cases. We refer to it as the density-matrix (DM) theory.

In view of the fact that the DM theory is an exact limit of quantum mechanics it is important to know its properties in some detail. Being an asymptotic theory it is clear that it does not encompass the same information as the full Hamiltonian at finite $Z$ and $B$. In particular, the DM theory does not capture exchange-correlation effects, and it is a theory of very strong fields in the sense that all electrons are confined to the lowest Landau band. These features should not be considered as a shortcoming of the DM theory, however. In fact, the hardest part of the derivation of the limit theorems in [4] is precisely to prove rigorously that contributions from exchange and higher Landau bands vanish in the limit considered. The DM theory should be judged on its own merits: It is enormously more simple numerically than the full quantum mechanical problem (2) and it is a well defined starting point for more refined approximations.

In the present contribution we report on a numerical study of the DM theory for atoms. We have computed ground-state energies and electronic density profiles over a wide range of parameters and compared them with results obtained by different methods. In particular, we compare the DM theory to the semiclassical theory that applies in region 3 , the simple density functional theory for region 5 , and also to other density functional $[16,17]$ and Hartree-Fock (HF) [18] calculations. The difference between DM and HF calculations of ground-state energies is less than $2 \%$ where data are available so that comparison can be made. This is remarkable in view of the fact that for standard Thomas-Fermi (TF) theory with $B=0$ the Scott term, which corrects for the rough treatment of the electrons close to the nucleus in TF theory, must be incorporated in order to achieve such a good numerical agreement, cf. [19]. Thus, at least at this field strength, DM theory is closer to HF theory than might have been expected. A more precise statement requires an analysis of the next to leading order terms in the asymptotic expansion of the ground-state energy. Such an analysis has yet to be carried out.

Another point where the DM theory differs from semiclassical theories is in the possibility of negative ionization. It is a general feature of Thomas-Fermi type theories, based on potential theoretical arguments, cf. [20], that the number of bound electrons never exceeds $Z$. For the quantum mechanical problem the meaning of this is simply that the binding energy of an excess electron must necessarily be of lower order in $Z$ than the ground-state energy. On the other hand, it is known that a magnetic field enhances binding, for instance, the Hamiltonian (1) with $N=Z+1$ has infinitely many bound states for $B \neq 0$ [21]. In the limit of extremely strong fields in region 5 the negative charge can even be as large as $2 Z$ [4]. The only rigorous results on the DM theory concern this extreme limit, but our numerical computations clearly show negative ionization that increases with $B$. It seems, however, that in order to approach the $2 Z$ value extremely strong fields are needed; even at fields as strong ${ }^{2}$ as $10^{18} \mathrm{G}$ the excess charge for iron is "only" about $23 \%$.

Our interest in negative ionization is also motivated by its relation to another question, the binding of atoms into molecules and chains. Although a rigorous mathematical theorem linking these two aspects of binding does not seem to exist, it is a fact that in regions $1-3$, i.e., for $B \ll Z^{3}$, molecular binding energies are vanishingly small compared to ground-state energies, whereas in region 5 binding becomes extremely strong: For a diatomic molecule the binding energy is six times the ground-state energy of an individual atom. The question whether iron is weakly or strongly bound at field strengths of the order $10^{12} \mathrm{G}$ has been controversial over the past 25 years. The best HF results [18] indicate weak or no binding, but the computations are difficult for they amount to subtracting one large number from another. It is decisive to treat the molecules and the individual atoms consistently by the same numerical methods so that unavoidable errors cancel as far as possible. Since the DM theory is numerically much simpler than HF theory it is easier to achieve this in the former and we plan to return to the binding question in a separate paper. The atomic computations presented here are a necessary preparation for the study of molecules and chains.

\section{THE DENSITY-MATRIX THEORY AND ITS LIMITING CASES}

The density-matrix theory $[6,4]$ is based on an energy functional that depends not simply on the electronic density, but rather on density matrices of a certain kind. To be more precise, the variable of the functional is a mapping $\Gamma$ : $\mathbf{r}_{\perp} \mapsto \Gamma_{\mathbf{r}_{\perp}}$ which, for each point $\mathbf{r}_{\perp}=(x, y)$ in the twodimensional plane perpendicular to the field, defines an integral kernel $\Gamma_{\mathbf{r}_{\perp}}\left(z, z^{\prime}\right)$ with $\int \Gamma_{\mathbf{r}_{\perp}}(z, z) d z<\infty$. Here $z$ is the one-dimensional spatial variable along the field. In a magnetic field of strength $B$ these kernels have to satisfy the condition

$$
0 \leqslant \iint \Gamma_{\mathbf{r}_{\perp}}\left(z, z^{\prime}\right) \psi(z)^{*} \psi\left(z^{\prime}\right) d z d z^{\prime} \leqslant \frac{B}{2 \pi} \int|\psi(z)|^{2} d z
$$

for all one-dimensional wave functions $\psi$ and all $\mathbf{r}_{\perp}$. The density in three-dimensional space corresponding to such a

\footnotetext{
${ }^{2}$ The computations at these extreme field strengths were carried out mainly to test the mathematical properties of DM theory. It is clear that doubts about the applicability of the nonrelativistic Hamiltonian (1) can be raised in such extreme fields, even for very heavy atoms.
} 
$\Gamma$ is $\rho_{\Gamma}(\mathbf{r})=\Gamma_{\mathbf{r}_{\perp}}(z, z)$ for $\mathbf{r}=\left(\mathbf{r}_{\perp}, z\right)$. The density-matrix functional for an atom with nuclear charge $Z$ is defined by

$$
\begin{aligned}
\mathcal{E}^{\mathrm{DM}}[\Gamma]= & -\int\left[\frac{\partial^{2} \Gamma_{\mathbf{r}_{\perp}}\left(z, z^{\prime}\right)}{\partial z^{\prime 2}}\right]_{z^{\prime}=z} d^{3} \mathbf{r}-Z \int \frac{\rho_{\Gamma}(\mathbf{r})}{|\mathbf{r}|} d^{3} \mathbf{r} \\
& +\frac{1}{2} \iint \frac{\rho_{\Gamma}(\mathbf{r}) \rho_{\Gamma}\left(\mathbf{r}^{\prime}\right)}{\left|\mathbf{r}-\mathbf{r}^{\prime}\right|} d^{3} \mathbf{r}^{\prime} d^{3} \mathbf{r}
\end{aligned}
$$

In the density matrix theory the electrostatic interactions are treated classically but the kinetic energy for the motion along the magnetic field is treated quantum mechanically by the $-\partial^{2} / \partial z^{2}$ term. In directions perpendicular to the field the motion is restricted by the "hard core" condition (4). This condition reflects the fact that the density of states per unit area for free electrons in the lowest Landau band is $B /(2 \pi)$. The functional (5) is plausible if one thinks of $\Gamma_{\mathbf{r}_{\perp}}\left(z, z^{\prime}\right)$ as an approximation to

$$
N \int \Psi_{0}\left(\mathbf{r}_{\perp}, z ; \mathbf{r}_{2}, \ldots, \mathbf{r}_{N}\right) \Psi_{0}^{*}\left(\mathbf{r}_{\perp}, z^{\prime} ; \mathbf{r}_{2}, \ldots, \mathbf{r}_{N}\right) \prod_{j=2}^{N} d^{3} \mathbf{r}_{j},
$$

where $\Psi_{0}$ is a normalized ground-state wave function. In the parameter region $B \gg Z^{4 / 3}$ the electrons are confined to the lowest Landau band, and the Pauli Hamiltonian $\{[\mathbf{p}+\mathbf{A}(\mathbf{r})] \cdot \boldsymbol{\sigma}\}^{2}$, restricted to the lowest Landau band, is precisely $-\partial^{2} / \partial z^{2}$.

The ground-state energy for $N$ electrons in DM theory is

$$
E^{\mathrm{DM}}(N, B, Z)=\inf \left\{\mathcal{E}^{\mathrm{DM}}[\Gamma]: \int \rho_{\Gamma}(\mathbf{r}) d^{3} \mathbf{r} \leqslant N\right\} .
$$

As shown in [4], Theorem 4.3, there is a unique minimizer for this variational problem, i.e.,

$$
E^{\mathrm{DM}}(N, B, Z)=\mathcal{E}^{\mathrm{DM}}\left[\Gamma_{N, B, Z}^{\mathrm{DM}}\right],
$$

with a unique $\Gamma_{N, B, Z}^{\mathrm{DM}}$ The corresponding density, $\rho_{N, B, Z}^{\mathrm{DM}}$ satisfies $\int \rho_{N, B, Z}^{\mathrm{DM}}=N$, if $N \leqslant N_{c}$, and $\int \rho_{N, B, Z}^{\mathrm{DM}}=N_{c}$, if $N>N_{c}$, where $N_{c} \geqslant Z$ is a number depending on $Z$ and $B$. As explained in the next section, the minimization problem (7) amounts to seeking at each $\mathbf{r}_{\perp}$ the lowest eigenvalues and eigenfunctions for a one-dimensional Schrödinger Hamiltonian $-\partial^{2} / \partial z+V_{\mathbf{r}_{\perp}}^{\mathrm{DM}}(z)$ where $V_{\mathbf{r}_{\perp}}^{\mathrm{DM}}$ is the self-consistent potential generated by the nucleus and $\rho_{N, B, Z}^{\mathrm{DM}}$.

The density matrix theory is in fact a two parameter theory with parameters $\lambda=N / Z$ and $\eta=B / Z^{3}$ due to the scaling relations

$$
E^{\mathrm{DM}}(N, B, Z)=Z^{3} E^{\mathrm{DM}}(\lambda, \eta, 1)
$$

and

$$
\rho_{N, B, Z}^{\mathrm{DM}}(\mathbf{r})=Z^{4} \rho_{\lambda, \eta, 1}^{\mathrm{DM}}(Z \mathbf{r}) .
$$

In particular, the ratio to $Z$ of the maximal number of electrons that a nucleus can bind in DM theory, $\lambda_{c}=N_{c} / Z$, is a function of $\eta$ alone.

The DM theory holds a special position in the study of the properties of matter in strong magnetic field because it pro-

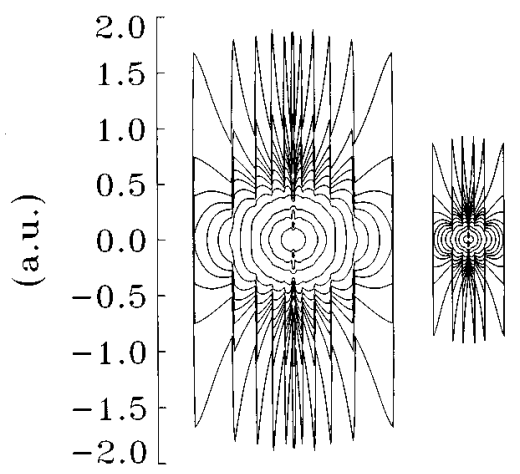

FIG. 1. Contour plots of the electronic density of iron atoms in DM theory for $B=10^{11} \mathrm{G}$ (left) and $B=10^{12} \mathrm{G}$ (right). The outermost contour encloses $99 \%$ of the negative charge, the next $90 \%$, then $80 \%$, etc., and the two innermost $5 \%$ and $1 \%$, respectively.

vides an asymptotically exact description of the quantum mechanical ground-state energy $E^{Q}$ and electron density $\rho^{Q}$ as $N, Z$, and $B$ tend to infinity with $N / Z$ fixed and $B / Z^{4 / 3} \rightarrow \infty$. The following theorems are proved in [4], Theorems 1.1 and 8.1 .

Theorem II.1. Let $N, Z \rightarrow \infty$ with $N / Z$ fixed. If $B / Z^{4 / 3} \rightarrow \infty$, then

$$
E^{Q}(N, B, Z) / E^{\mathrm{DM}}(N, B, Z) \rightarrow 1 .
$$

Theorem II.2. Let $N, Z$, and $B \rightarrow \infty$ with $N / Z=\lambda$ and $B / Z^{3}=\eta$ fixed. Then

$$
Z^{-4} \rho_{N, B, Z}^{Q}\left(Z^{-1} \mathbf{r}\right) \rightarrow \rho_{\lambda, \eta, 1}^{\mathrm{DM}}(\mathbf{r})
$$

in the sense of convergence of distributions.

The shape of atoms in DM theory is discussed in Sec. IV in connection with Figs. 1 and 2. It should be kept in mind that by the limit theorems II.1 and II.2 the DM theory is a theory of heavy atoms. We have chosen iron with $Z=26$ as our reference because of its astrophysical importance. By the scaling relations (9) and (10) it is simple to transform the results to other values of $Z$. As seen from the figures, the atom is approximately spherical when the magnetic field is not too strong ( $\leqslant 10^{11} \mathrm{G}$ for iron), but becomes increasingly

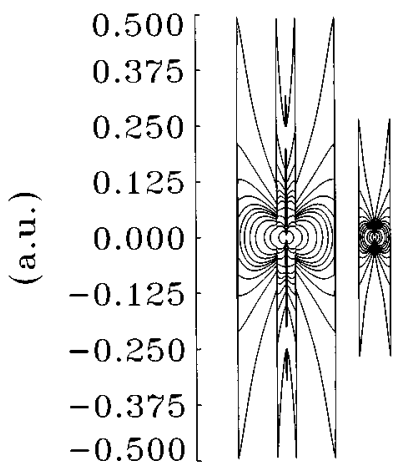

FIG. 2. Contour plots of the electron density of iron atoms in DM theory for $B=10^{13} \mathrm{G}$ (left) and $B=10^{14} \mathrm{G}$ (right). The contours are drawn in the same way as in Fig. 1 . At $B=10^{14}$ the DM model has simplified and the density is described by the SS functional (12). 
elongated as the field goes up. In fact, as shown in [4], the limiting cases $\eta \rightarrow 0$ and $\eta \rightarrow \infty$ of the DM theory can be described by simpler theories that we now review briefly, referring to $[4,5]$ for details.

The weak field limit, $\eta \rightarrow 0$, is the Thomas-Fermi theory for atoms in strong magnetic fields, where only the lowest Landau band is taken into account (as in DM theory). This theory was introduced by Kadomtsev [7] and studied further in a number of publications, see [5] for a list of references. In $[4,5]$ it is called the STF theory. The density functional is

$$
\begin{aligned}
\mathcal{E}^{\mathrm{STF}}[\rho]= & \frac{4 \pi^{4}}{3 B^{2}} \int \rho(\mathbf{r})^{3} d^{3} \mathbf{r}-Z \int \frac{\rho(\mathbf{r})}{|\mathbf{r}|} d^{3} \mathbf{r} \\
& +\frac{1}{2} \iint \frac{\rho(\mathbf{r}) \rho\left(\mathbf{r}^{\prime}\right)}{\left|\mathbf{r}-\mathbf{r}^{\prime}\right|} d^{3} \mathbf{r} d^{3} \mathbf{r}^{\prime}
\end{aligned}
$$

The precise connection between DM and STF theory is given in [4], Eq. (8.11); if $E^{\mathrm{STF}}(N, B, Z)$ denotes the infimum of (13) with subsidiary condition $\int \rho \leqslant N$, then

$$
\lim _{\eta \rightarrow 0} E^{\mathrm{DM}}(\lambda, \eta, 1) / \eta^{2 / 5}=E^{\mathrm{STF}}(\lambda, 1,1) .
$$

In STF theory, atoms are spherical with a finite radius $\sim Z^{-1 / 3}\left(B / Z^{4 / 3}\right)^{-2 / 5}$.

In the opposite parameter regime, more precisely for $\eta$ larger than a certain critical value, $\eta_{c}$, DM theory also reduces to a density functional theory. The value of $\eta_{c}$ depends on $\lambda$; for $\lambda=1$ we find $\eta_{c}=0.148$, which for $Z=26$ corresponds to $B=2.44 \times 10^{13} \mathrm{G}$. The energy functional appropriate for such superstrong (SS) fields is

$$
\begin{aligned}
\mathcal{E}^{S S}[\rho]= & \int[\partial \sqrt{\rho} / \partial z]^{2} d^{3} \mathbf{r}-Z \int \frac{\rho(\mathbf{r})}{|\mathbf{r}|} d^{3} \mathbf{r} \\
& +\frac{1}{2} \iint \frac{\rho(\mathbf{r}) \rho\left(\mathbf{r}^{\prime}\right)}{\left|\mathbf{r}-\mathbf{r}^{\prime}\right|} d^{3} \mathbf{r} d^{3} \mathbf{r}^{\prime}
\end{aligned}
$$

with the subsidiary conditions

$$
\begin{gathered}
\int \rho(\mathbf{r}) d^{3} \mathbf{r} \leqslant N \\
\int \rho(\mathbf{r}) d z \leqslant B /(2 \pi) \text { for all } \mathbf{r}_{\perp} .
\end{gathered}
$$

In fact, for $\eta \geqslant \eta_{c}$, the minimizer of (5) has the form

$$
\Gamma_{\mathbf{r}_{\perp}}^{\mathrm{DM}}\left(z, z^{\prime}\right)=\sqrt{\rho^{\mathrm{DM}}\left(\mathbf{r}_{\perp}, z\right)} \sqrt{\rho^{\mathrm{DM}}\left(\mathbf{r}_{\perp}, z^{\prime}\right)},
$$

and (5) evaluated for $\Gamma^{\mathrm{DM}}$ is the same as (15) evaluated for $\rho^{\mathrm{DM}}$. Atoms in SS theory have the form of a thin cylinder with axis in the direction of the magnetic field and with a cone-shaped region essentially cut out of its interior. The radius is finite, $R=\sqrt{2 Z / B}$. The extension along the field is infinite, but the bulk of the electrons is confined within a distance $\sim Z^{-1}\left[\ln \left(B / Z^{3}\right)\right]^{-1}$ from the nucleus.

An even greater simplification occurs in the extreme limit $\eta \rightarrow \infty$, which we refer to as the hyperstrong (HS) case. In this limit the atom becomes effectively one dimensional and is described by a functional that can be minimized in closed form. This functional is

$$
\mathcal{E}^{\mathrm{HS}}[\rho]=\int[\partial \sqrt{\rho} / \partial z]^{2} d z-\rho(0)+\int \rho(z)^{2} d z
$$

where $\rho(z)$ is a one-dimensional density and the subsidiary condition is

$$
\int \rho(z) d z \leqslant \lambda=N / Z
$$

The connection between the SS and HS theories is as follows. Let $E^{\mathrm{SS}}(N, B, Z)$ denote the minimum of (15) with the subsidiary conditions (16), and let $E^{\mathrm{HS}}(\lambda)$ denote the minimum of (18) with the subsidiary condition (19). Let $L(\eta)$ be the solution to the equation

$$
(\eta / 2)^{1 / 2}=L(\eta) \sinh [L(\eta) / 2]
$$

Then we have

$$
E^{\mathrm{SS}}(N, B, Z)=Z^{3} L(\eta)^{2} E^{\mathrm{HS}}(\lambda)+Z^{3} O(L(\eta)) .
$$

There is also a corresponding connection between the minimizing densities, $\rho_{N, B, Z}^{\mathrm{SS}}(\mathbf{r})$ and $\rho_{\lambda}^{\mathrm{HS}}(z)$, for the two theories. Namely,

$$
\left[Z^{2} L(\eta)\right]^{-1} \int \rho_{N, B, Z}^{\mathrm{SS}}\left(\mathbf{r}_{\perp},[Z L(\eta)]^{-1} z\right) d^{2} \mathbf{r}_{\perp} \rightarrow \rho_{\lambda}^{\mathrm{HS}}(z)
$$

(in the sense of distributions).

The function $L(\eta)$ behaves like $\ln \eta$ for large $\eta$, so the convergence of $E^{\mathrm{SS}}$ to $E^{\mathrm{HS}}$ is rather slow. The main interest in the HS theory is that $\rho_{\lambda}^{\mathrm{HS}}(z)$ and $E^{\mathrm{HS}}(\lambda)$ can be explicitly computed: Writing $\rho_{\lambda}^{\mathrm{HS}}$ as $\left[\psi_{\lambda}^{\mathrm{HS}}\right]^{2}$ one has

$$
\begin{gathered}
\psi_{\lambda}^{\mathrm{HS}}(z)=\frac{\sqrt{2}(2-\lambda)}{4 \sinh \left[\frac{1}{4}(2-\lambda)|z|+c\right]} \text { for } \lambda<2, \\
\psi_{\lambda}^{\mathrm{HS}}(z)=\sqrt{2}(2+|z|)^{-1} \quad \text { for } \lambda \geqslant 2,
\end{gathered}
$$

where $\tanh c=(2-\lambda) / 2$. Moreover, ${ }^{3}$

$$
E^{\mathrm{HS}}(\lambda)=-\frac{1}{4} \lambda+\frac{1}{8} \lambda^{2}-\frac{1}{48} \lambda^{3}
$$

for $\lambda \leqslant 2$ and $E^{\mathrm{HS}}(\lambda)=E^{\mathrm{HS}}(2)=-1 / 6$ for $\lambda>2$. Thus $\lambda_{c}=2$ in HS theory. Equation (21) is essentially the statement of Theorem 3.5 in [4], but with one refinement: By replacing $\ln \eta$ in that theorem by $L(\eta)$ one obtains a neater estimate for the error term. The proof of (21) and (22), which follows closely the proof of Theorem 3.5 in [4], is given in the Appendix.

\footnotetext{
${ }^{3} \mathrm{We}$ recall that our energy unit is $54.4 \mathrm{eV}$.
} 


\section{NUMERICAL MINIMIZATION OF THE DENSITY-MATRIX FUNCTIONAL}

In this section we describe in some detail the numerical methods used to study the DM theory. The task is to minimize numerically the density-matrix functional (5) under the constraints (4) and $N=\int \rho_{\Gamma}(\mathbf{r}) d^{3} \mathbf{r}$. The density matrix can be expressed in the form

$$
\Gamma_{\mathbf{r}_{\perp}}\left(z, z^{\prime}\right)=\sum_{j=1}^{\infty} \lambda_{j}^{\mathbf{r}_{\perp}} \phi_{j}^{\mathbf{r}_{\perp}}\left(z^{\prime}\right)^{*} \phi_{j}^{\mathbf{r}_{\perp}}(z),
$$

where $\phi_{j}^{\mathbf{r}_{\perp}}(z)$ is an orthonormal basis in the Hilbert space $L^{2}(\mathbf{R}, d z)$ of one-dimensional wave functions for each $\mathbf{r}_{\perp}$ and $0 \leqslant \lambda_{j}^{\mathbf{r}_{\perp}} \leqslant B /(2 \pi)$, by condition (4). For the minimizer $\Gamma^{\mathrm{DM}}$ it turns out that

$$
\lambda_{j}^{\mathbf{r}_{\perp}}=\left\{\begin{array}{cc}
B / 2 \pi & \text { if } j \leqslant j_{c}^{\mathbf{r}_{\perp}} \\
0 & \text { if } j>j_{c}^{\mathbf{r}_{\perp}}
\end{array}\right.
$$

with $j \leqslant j_{c}^{\mathbf{r}_{\perp}}<\infty$. In fact, $j \leqslant j_{c}^{\mathbf{r}_{\perp}} \leqslant j_{c}$ with a $j_{c}<\infty$ that is independent of $\mathbf{r}_{\perp}$ (but depends on $B$ ), cf. [4], p. 553. The functions $\phi_{j}^{\mathbf{r}_{\perp}}$ satisfy the one-dimensional Schrödinger equation

$$
\left[-\frac{\partial^{2}}{\partial z^{2}}-\frac{Z}{|\mathbf{r}|}+\int \frac{\rho_{\Gamma}\left(\mathbf{r}^{\prime}\right)}{\left|\mathbf{r}-\mathbf{r}^{\prime}\right|} d^{3} \mathbf{r}^{\prime}\right] \phi_{j}^{\mathbf{r}_{\perp}}(z)=\epsilon_{j}^{\mathbf{r}_{\perp}} \phi_{j}^{\mathbf{r}_{\perp}}(z)
$$

and for each $(N, Z, B)$ there exists a unique $\mu^{\mathrm{DM}}$ such that $j \leqslant j_{c}^{\mathbf{r}_{\perp}}$ if and only if $\boldsymbol{\epsilon}_{j}^{\mathbf{r}_{\perp}} \leqslant \mu^{\mathrm{DM}}$, and (26) is the solution of the minimization problem. Our strategy is to minimize the density matrix functional (5) by iteratively solving the set of nonlinear eigenvalue equations $(28)$ and determining $\mu^{\mathrm{DM}}$ such that the constraint $\int \rho_{\Gamma}=N$ is satisfied. The eigenvalue equations are invariant with respect to rotation around the $z$ axis. Hence they depend only on $\left|\mathbf{r}_{\perp}\right|$, but even with this reduction (28) yields an infinite number of eigenvalue equations, one for each value of $\left|\mathbf{r}_{\perp}\right|$. We reduce them to a finite number by making the $\left|\mathbf{r}_{\perp}\right|$ axis discrete. The DM atom has a finite radius $R \leqslant R_{0}=\sqrt{2 N / B}$. We therefore only have to consider the eigenvalue equations for which $\left|\mathbf{r}_{\perp}\right| \leqslant R$. Let $N_{\perp}$ be the number of eigenvalue equations we choose to work with. Let

$$
\Delta_{\perp}=\frac{R_{0}}{N_{\perp}-1} .
$$

We solve (28) at the $N_{\perp}$ points $n \Delta_{\perp}, n=1, \ldots, N_{\perp}$, on the $\left|\mathbf{r}_{\perp}\right|$ axis. Let

$$
\theta_{n}^{\perp}(r)= \begin{cases}1 & \text { if } r \in\left((n-1) \Delta_{\perp}, n \Delta_{\perp}\right] \\ 0 & \text { otherwise. }\end{cases}
$$

We minimize the density-matrix functional (5) with density matrices of the form

$$
\Gamma_{\mathbf{r}_{\perp}}\left(z^{\prime}, z\right)=\sum_{j=1}^{\infty} \sum_{n=1}^{N_{\perp}} \lambda_{j}^{n \Delta_{\perp}} \phi_{j}^{n \Delta_{\perp}}\left(z^{\prime}\right)^{*} \phi_{j}^{n \Delta_{\perp}}(z) \theta_{n}^{\perp}\left(\left|\mathbf{r}_{\perp}\right|\right) .
$$

Let

$$
h_{n}=-\frac{\partial^{2}}{\partial z^{2}}-\frac{Z}{\sqrt{\left(n \Delta_{\perp}\right)^{2}+z^{2}}}
$$

and let $\hat{\psi}_{i}^{n}$ denote the eigenfunctions of $h_{n}$ and $\hat{\mu}_{i}^{n}$ the corresponding eigenvalues, so that $h_{n} \hat{\psi}_{i}^{n}=\hat{\mu}_{i}^{n} \hat{\psi}_{i}^{n}$. We express $\phi_{j}^{n \Delta_{\perp}}(z)$ in terms of approximate eigenfunctions of $h_{n}$ which correspond to the $N_{b}$ lowest eigenvalues. Hence we write

$$
\phi_{j}^{n \Delta_{\perp}}(z)=\sum_{i=1}^{N_{b}} c_{j i}^{n} \psi_{i}^{n}(z)
$$

where $\psi_{i}^{n}$ is an approximation for $\hat{\psi}_{i}^{n}$. We determine approximations for the basis functions $\hat{\psi}_{i}^{n}$ and their eigenvalues $\hat{\mu}_{i}^{n}$ by the method of finite elements (FEM) for eigenvalue problems, dividing the interval $\left[-z_{m}, z_{m}\right], z_{m}>0$ into elements $^{4}$ and choosing a polynomial basis of degree 5 within each, cf. [22]. Let these solutions be $\psi_{i}^{n}(z)$ where we induce the boundary condition that $\psi_{i}^{n}\left( \pm z_{m}\right)=0$. The eigenvalue corresponding to $\psi_{i}^{n}(z)$ is denoted by $\mu_{i}^{n}$. Let $N_{\|}$be the number of samples of $\psi_{i}^{n}(z)$ values we choose to work with along the $z$ axis and define

$$
\Delta_{\|}=\frac{2 z_{m}}{N_{\|}-1} .
$$

Then the samples we work with are $\psi_{i}^{n}\left((l-1 / 2) \Delta_{\|}-z_{m}\right)$, $l=1, \ldots, N_{\|}$. We use the values $\mu_{i}^{n}$ as approximations for $\hat{\mu}_{i}^{n}$. This basis is chosen because it is expected to be close to the solutions $\phi_{j}^{n \Delta_{\perp}}(z)$ and as such is a natural starting point for the self-consistent iterations. We have now defined the set over which we numerically minimize (5).

We solve the set of nonlinear equations (28) in a selfconsistent manner iteratively. We define the chain of potentials

$$
V_{1}^{(k)}(\mathbf{r})=(1-\alpha) V_{1}^{(k-1)}(\mathbf{r})+\alpha V_{0}^{(k-1)}(\mathbf{r})
$$

for $k>0$ and $V_{1}^{(0)}=0, \alpha \in(0,1] .^{5}$ Let $\phi_{j}^{n \Delta_{\perp},(k)}(z)$ be the eigenfunctions of the operator

$$
H^{n \Delta_{\perp},(k)}=h_{n}+V_{1}^{(k)}(\mathbf{r}),
$$

with

$$
V_{0}^{(k)}(\mathbf{r})=\int \frac{\Gamma_{x_{\perp}^{\prime}}^{(k)}\left(z^{\prime}, z^{\prime}\right)}{\left|\mathbf{r}-\mathbf{r}^{\prime}\right|} d^{3} \mathbf{r}^{\prime}
$$

where

\footnotetext{
${ }^{4}$ The number of elements we use are 50-60, and their width varies such that the smallest elements are closest to the origin.

${ }^{5}$ Self-consistent iterations of the kind discussed here are in general not convergent. The coefficient $(1-\alpha)$ acts as a damping factor on the iterations. The value of $\alpha$ is in practice chosen by trial and error as high as possible without inducing instability. That way the iterations converge as fast as is possible.
} 


$$
\begin{aligned}
\Gamma_{\mathbf{r}_{\perp}}^{(k)}\left(z, z^{\prime}\right)= & \sum_{j=1}^{N_{b}} \sum_{n=1}^{N_{\perp}} \lambda_{j}^{n \Delta_{\perp},(k)} \phi_{j}^{n \Delta_{\perp},(k)}\left(z^{\prime}\right)^{*} \\
& \times \phi_{j}^{n \Delta_{\perp},(k)}(z) \theta_{n}^{\perp}\left(\left|\mathbf{r}_{\perp}\right|\right) .
\end{aligned}
$$

Note that $\phi_{j}^{n \Delta_{\perp},(0)}(z)=\psi_{j}^{n}(z)$. With an appropriate choice of $\alpha$ (we use $\alpha \in[0.01,0.1]$ ) the sequence $\phi_{j}^{n \Delta_{\perp},(k)}(z)$ turns out to be convergent and

$$
\lim _{k \rightarrow \infty} \phi_{j}^{n \Delta_{\perp},(k)}(z)=\phi_{j}^{n \Delta_{\perp}}(z)
$$

in $L^{2}\left(\left[-z_{m}, z_{m}\right], d z\right)$. This defines our self-consistent iteration scheme. To be consistent with the discrete form of (38) we work with the potentials $V_{1}^{(k)}$ and $V_{0}^{(k)}$ of the form

$$
V\left(z, \mathbf{r}_{\perp}\right)=\sum_{l=1}^{N_{\|}} \sum_{n=1}^{N_{\perp}} V_{l n} \theta_{l}^{\|}(z) \theta_{n}^{\perp}\left(\mathbf{r}_{\perp}\right),
$$

where

$$
\theta_{l}(z)= \begin{cases}1 & \text { if } z \in\left((l-1) \Delta_{\|}-z_{m}, l \Delta_{\|}-z_{m}\right] \\ 0 & \text { otherwise. }\end{cases}
$$

To determine (37) we calculate the boundary values on the $N_{\perp} \times N_{\|}$grid we work on by direct integration,

$$
\begin{aligned}
\int \frac{\Gamma_{x_{\perp}^{\prime}}^{(k)}\left(z^{\prime}, z^{\prime}\right)}{\left|\mathbf{r}-\mathbf{r}^{\prime}\right|} d^{3} \mathbf{r}^{\prime}= & \frac{2}{\sqrt{r}} \int_{0}^{\infty} d r^{\prime} \sqrt{r^{\prime}} \Gamma_{x_{\perp}^{\prime}}^{(k)}\left(z^{\prime}, z^{\prime}\right) \\
& \times Q_{-1 / 2}\left(\frac{r^{2}+r^{\prime 2}}{2 r r^{\prime}}\right),
\end{aligned}
$$

where $Q_{\nu-1 / 2}$ is an associated Legendre function. The direct integration of (37) is very slow but it is, on the other hand, very accurate. To determine $V_{0}^{(k)}$ at interior grid points faster numerically we do the following: We note that (37) is the solution of the Poisson equation

$$
\nabla^{2} V_{0}^{(k)}=\Gamma_{\mathbf{r}_{\perp}}^{(k)}
$$

With the boundary values determined by the direct integration we use standard five point difference approximation to

TABLE I. Ground-state energy (in $\mathrm{keV}$ ) of iron atoms $(Z=26)$ as a function of the magnetic field $B$ (in $\mathbf{G}$ ) and the ratio $\lambda=N / Z$ of electron number to nuclear charge.

\begin{tabular}{lccccc}
\hline \hline$\backslash B$ & $10^{10}$ & $10^{11}$ & $10^{12}$ & $10^{13}$ & $10^{14}$ \\
\hline 0.1 & -3.202 & -8.188 & -20.80 & -52.58 & -122.4 \\
0.2 & -4.753 & -12.07 & -30.56 & -77.56 & -185.2 \\
0.3 & -5.838 & -14.80 & -37.47 & -95.04 & -230.2 \\
0.4 & -6.670 & -16.88 & -42.64 & -108.0 & -264.0 \\
0.5 & -7.277 & -18.41 & -46.56 & -117.9 & -289.4 \\
0.6 & -7.744 & -19.58 & -49.53 & -125.2 & -309.3 \\
0.7 & -8.102 & -20.42 & -51.73 & -130.6 & -323.7 \\
0.8 & -8.321 & -21.01 & -53.12 & -134.1 & -333.5 \\
0.9 & -8.475 & -21.41 & -53.85 & -136.6 & -339.9 \\
1.0 & -8.521 & -21.47 & -54.38 & -137.8 & -342.7 \\
\hline \hline
\end{tabular}

the Laplacian in order to determine $V_{0}^{(k)}$ at the interior points. We solve the finite difference scheme by simultaneous overrelaxation. We find that an over-relaxation coefficient of 1.8 yields fast convergence for this problem for our choice of $N_{\perp}$ and $N_{\|}$. When we use the finite difference scheme we predetermine $V_{0}^{(k)}$ at a few interior grid points by direct integration. We then perform the over-relaxation iterations until we obtain agreement with the predetermined values up to a desired accuracy. We choose to iterate until the first five digits of the solution are identical to all the predetermined values.

Now we are ready to go through one iteration of the selfconsistent iteration scheme in detail. Let us consider the $k$ th step in the iteration. At the start of this step we know

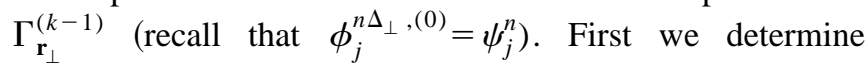
$V_{0}^{(k-1)}$ and then $V_{1}^{(k)}$ according to the scheme described above. We next determine the matrix elements

$$
\begin{aligned}
H_{l m}^{n \Delta_{\perp},(k)} & =\int \psi_{l}^{n} H^{n \Delta_{\perp},(k)} \psi_{m}^{n} d z \\
& =\delta_{l m} \widetilde{\epsilon}_{l}^{n}+\int \psi_{l}^{n} V_{1}^{(k)}(\mathbf{r}) \psi_{m}^{n} d z
\end{aligned}
$$

for each $n=1, \ldots, N_{\perp}$. Now the eigenvectors of the matrices $H_{l m}^{n \Delta_{\perp},(k)}$ correspond to the coefficients $c_{j i}^{n,(k)}$ in

$$
\phi_{j}^{n \Delta_{\perp},(k)}(z)=\sum_{i=1}^{N_{b}} c_{j i}^{n,(k)} \psi_{i}^{n}(z)
$$

and we denote their corresponding eigenvalues by $\epsilon_{j}^{n \Delta_{\perp},(k)}$. That is,

$$
H^{n \Delta_{\perp},(k)} \phi_{j}^{n \Delta_{\perp},(k)}(z)=\epsilon_{j}^{n \Delta_{\perp},(k)} \phi_{j}^{n \Delta_{\perp},(k)}(z)
$$

To determine the eigenvectors and eigenvalues of the matrices $H_{l m}^{n \Delta_{\perp},(k)}$ we use the eigenroutines from [23]. Finally, at the end of the iteration step we determine $\mu^{\mathrm{DM},(k)}$ such that $\int \Gamma_{\mathbf{r}_{\perp}}^{(k)}(z, z) d^{3} \mathbf{r}=N$. We are then ready for the next step of the iteration.

We continue the iterations until $\mathcal{E}^{\mathrm{DM}}\left(\Gamma^{(k)}\right)$ "stops", changing. More precisely we choose to stop when the change in $\mathcal{E}^{\mathrm{DM}}\left(\Gamma^{(k)}\right)$ between iterations is in the seventh digit.

We calculate with $N_{\perp}=101, \quad N_{\|}=201-301$, and $N_{b}=30-60$. We chose $z_{m}$ in such a way that $2 z_{m}$ is at least three times longer than the length of the atom the calculation

TABLE II. The ratio $\lambda_{c}=N_{c} / Z$ of the maximal negative charge to nuclear charge as a function of $B$ (in $\mathrm{G}$ ) for iron $(Z=26)$.

\begin{tabular}{lccc}
\hline \hline$B$ & $\lambda_{c}$ & $B$ & $\lambda_{c}$ \\
\hline $10^{10}$ & 1.020 & $10^{15}$ & 1.110 \\
$10^{11}$ & 1.026 & $10^{16}$ & 1.153 \\
$10^{12}$ & 1.035 & $10^{17}$ & 1.184 \\
$10^{13}$ & 1.043 & $10^{18}$ & 1.232 \\
$10^{14}$ & 1.061 & & \\
\hline \hline
\end{tabular}


yields. We obtained this criterion by increasing $z_{m}$ until the ground-state energy we obtained became stable in the first six digits.

To calculate the energy $\mathcal{E}^{\mathrm{DM}}$ we note that

$$
\begin{aligned}
E^{\prime}: & =\sum_{j} \int \lambda_{j}^{\mathbf{r}_{\perp}} \boldsymbol{\epsilon}_{j}^{\mathbf{r}_{\perp}} d^{2} \mathbf{r}_{\perp}=\sum_{j=1}^{N_{b}} \sum_{n=1}^{N_{\perp}} 2 \pi n \Delta_{\perp}^{2} \lambda_{j}^{n \Delta_{\perp}} \epsilon_{j}^{n \Delta_{\perp}} \\
& =K^{\mathrm{DM}}-A^{\mathrm{DM}}+2 R^{\mathrm{DM}} .
\end{aligned}
$$

Here $K^{\mathrm{DM}}, A^{\mathrm{DM}}$, and $R^{\mathrm{DM}}$ are, respectively, the kinetic, attractive, and repulsive parts of $\mathcal{E}^{\mathrm{DM}}$. With our choice of basis $\psi_{i}^{n}$ and $V(\mathbf{r})$ as the attractive potential we have

$$
\begin{aligned}
E^{\prime \prime}:= & \int\left[-\frac{\partial^{2} \Gamma_{\mathbf{r}_{\perp}}\left(z, z^{\prime}\right)}{\partial z^{\prime 2}}+V(\mathbf{r}) \Gamma_{\mathbf{r}_{\perp}}(z, z)\right]_{z^{\prime}=z} d^{3} \mathbf{r} \\
= & \sum_{j=1}^{N_{b}} \int \lambda_{j}^{\mathbf{r}_{\perp}} \boldsymbol{\phi}_{j}^{\mathbf{r}_{\perp}}(z)\left[-\frac{\partial^{2}}{\partial z^{2}}+V(\mathbf{r})\right] \boldsymbol{\phi}_{j}^{\mathbf{r}_{\perp}}(z) d^{3} \mathbf{r} \\
= & \sum_{j=1} \sum_{l=1}^{N_{b}} \sum_{k=1}^{N_{b}} \sum_{n=1}^{N_{\mathbf{r}_{\perp}}} \int \lambda_{j}^{n \Delta_{\perp}} c_{j l}^{n} c_{j k}^{n} \theta_{n}^{\perp}\left(\left|\mathbf{r}_{\perp}\right|\right) \\
& \times \psi_{l}^{n}(z) h_{n} \psi_{k}^{n}(z) d^{3} \mathbf{r} \\
= & \sum_{j=1}^{N_{b}} \sum_{l=1}^{N_{b}} \sum_{k=1}^{N_{b}} \sum_{n=1}^{N_{\mathbf{r}_{\perp}}} 2 \pi n \Delta_{\perp}^{2} \lambda_{j}^{n \Delta_{\perp}} c_{j l}^{n} c_{j k}^{n} \delta_{l k} \mu_{l}^{n} \\
= & \sum_{j=1}^{N_{b}} \sum_{l=1}^{N_{b}} \sum_{n=1}^{N_{\perp}} 2 \pi n \Delta_{\perp}^{2} \lambda_{j}^{n \Delta_{\perp}}\left(c_{j l}^{n}\right)^{2} \mu_{l}^{n}=K^{\mathrm{DM}}-A^{\mathrm{DM}} .
\end{aligned}
$$

Since

$$
A^{\mathrm{DM}}=-\int V(\mathbf{r}) \Gamma_{\mathbf{r}_{\perp}}(z, z) d^{3} \mathbf{r}
$$

we obtain

$$
\begin{gathered}
E^{\mathrm{DM}}=\frac{1}{2}\left(E^{\prime}+E^{\prime \prime}\right), \\
K^{\mathrm{DM}}=E^{\prime \prime}+A^{\mathrm{DM}}, \\
R^{\mathrm{DM}}=\frac{1}{2}\left(E^{\prime}-E^{\prime \prime}\right) .
\end{gathered}
$$

This is how we evaluate the ground-state energy and the terms it is composed of. Regarding the accuracy in the evaluation of $K^{\mathrm{DM}}$, one should be aware of the fact that $A^{\mathrm{DM}}$ is in
TABLE IV. Comparison of the ground-state energy (in keV) of iron atoms in DM theory, STF theory, and HS theory at various field strengths $B$ (in G). See also Fig. 3.

\begin{tabular}{cccc}
\hline \hline$B$ & $E^{\mathrm{DM}}$ & $E^{\mathrm{STF}}$ & $E^{\mathrm{HS}}$ \\
\hline $10^{11}$ & -21.47 & -21.57 & \\
$10^{12}$ & -54.37 & -54.07 & \\
$10^{13}$ & -137.8 & -135.8 & \\
$10^{14}$ & -342.7 & -341.2 & -0.3346 \\
$10^{15}$ & -786.3 & -857.0 & -535.3 \\
$10^{16}$ & -1623 & -2153 & -1797 \\
$10^{17}$ & -3065 & -5405 & -3913 \\
$10^{18}$ & -5264 & -13583 & \\
\hline \hline
\end{tabular}

general a lot larger than $K^{\mathrm{DM}}$. In the case of the STF theory $A^{\mathrm{STF}}=15 K^{\mathrm{STF}}$, which is the low magnetic field strength limit for the DM theory. Therefore a slight relative error in $A^{\mathrm{DM}}$ yields a much larger relative error in $K^{\mathrm{DM}}$. Based on the information given above, we estimate the numerical error of the scheme to be about \pm 1 in the fourth digit of the groundstate energy. We therefore show the first four digits when we present our results of the energy.

The estimate of $\lambda_{c}$ is done in the following way. $E^{\mathrm{DM}}(\lambda, \eta)$ is calculated as a function of $\lambda$ which is a strictly convex function in our approximation, due to the finite box. The minimum of this function then determines $\lambda_{c}$. However, the function is extremely flat around the minimum so it is difficult to determine its position. We make a linear approximation of $E^{\mathrm{DM}}(\lambda, \eta)$ using two close lying points below the minimum. The value thus obtained gives a lower bound on $\lambda_{c}$ close to the true value.

\section{ATOMIC PROPERTIES IN DM THEORY}

The results of our numerical computations are presented in Tables I-VI and illustrated in Figs. 1-5. As remarked before, iron is of special importance in astrophysical context, and for this reason we state our results for the reference value $Z=26$. The scaling relations (9) and (10) allow an easy transformation to other values. Moreover, in this section magnetic fields are measured in gauss and energies in keV to facilitate comparison with astrophysical data and other computations. To transform into the units in which the original Hamiltonian (1) is written it should be kept in mind that there the energy unit is $54.4 \mathrm{eV}$ and the unit for magneticfield strength is $9.40 \times 10^{9} \mathrm{G}$. The dimensionless parameter $\eta=B / Z^{3}$ has for $Z=26$ and $B=10^{12} \mathrm{G}$ the value $6.053 \times 10^{-3}$. This may seem small for region 4 , but as discussed in [5], the relevant semiclassical parameter is really

TABLE III. The binding energy at maximal negative ionization in DM theory.

\begin{tabular}{cccc}
\hline \hline$B$ & {$\left[E^{\mathrm{DM}}\left(\lambda_{c}\right)-E^{\mathrm{DM}}(1)\right] / E^{\mathrm{DM}}(1)$} & $B$ & {$\left[E^{\mathrm{DM}}\left(\lambda_{c}\right)-E^{\mathrm{DM}}(1)\right] / E^{\mathrm{DM}}(1)$} \\
\hline $10^{10}$ & 0.0011 & $10^{15}$ & 0.0073 \\
$10^{11}$ & 0.0008 & $10^{16}$ & 0.0123 \\
$10^{12}$ & 0.0035 & $10^{17}$ & 0.0232 \\
$10^{13}$ & 0.0038 & $10^{18}$ & 0.0203 \\
$10^{14}$ & 0.0050 & & \\
\hline \hline
\end{tabular}




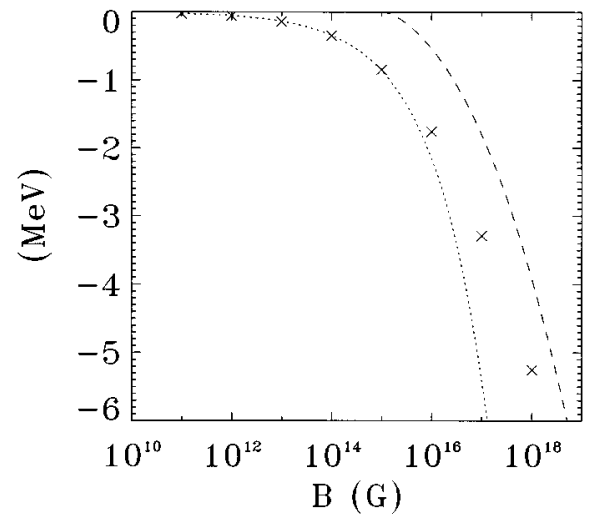

FIG. 3. The ground-state energy of iron atoms as a function of the magnetic field strength $B$ in DM theory (crosses), STF theory (short dashes), and HS theory (long dashes).

$\eta^{1 / 5}$ and at the quoted values of $Z$ and $B$ we have $\eta^{1 / 5}=0.36$.

Figures 1 and 2 show contour plots of the electronic densities of iron atoms with $N=Z$ according to DM theory at four different field strengths, ranging from $10^{11}$ to $10^{14} \mathrm{G}$. It is evident that in the weakest field the bulk of the electrons is spherically distributed around the nucleus. With increasing field strength the sphericity gets more and more distorted. The atom is composed of cylindrical shells that decrease in number as the field goes up. Between $10^{13}$ and $10^{14} \mathrm{G}$ a transition to a cylindrical shape with a single shell takes place. The number of shells corresponds to the number of eigenfunctions of the one-dimensional Schrödinger operators $-\partial^{2} / \partial z^{2}+V_{\mathbf{r}_{\perp}}^{\mathrm{DM}}(z)$ that contribute to the density matrix in the sum (26). The critical value $\eta_{c}$ at which this number has dropped to one was determined numerically for $\lambda=1$ to be $\eta_{c}=0.148$. This corresponds to $B=2.44 \times 10^{13} \mathrm{G}$ for $Z=26$.

In Table I the ground-state energy (7) of iron is shown as a function of the field strength for $B$ between $10^{10}$ and $10^{14} \mathrm{G}$ and for various values of the ratio $\lambda=N / Z$ of electron number to nuclear charge. A comparison of some of these values with results obtained by other methods is given in the next section.

Table II shows the results for $\lambda_{c}$ as a function of $B$ in DM theory. At the extremely strong field of $10^{18} \mathrm{G}$, corresponding to $\eta^{1 / 5}=5.7$, one finds $\lambda_{c}=1.232$, which is still quite far from the HS value $\lambda_{c}=2$. However, compared with ThomasFermi theories, where $\lambda_{c}$ is always 1 [20], the negative ionization is noticeable even for the weaker fields. The value $\lambda_{c}=1.046$ for iron at $B=10^{13} \mathrm{G}$ corresponds to an excess negative charge of 1.2 electrons. The binding energy of the excess charge in DM theory is shown in Table III.

As remarked at the end of Sec. III a precise determination

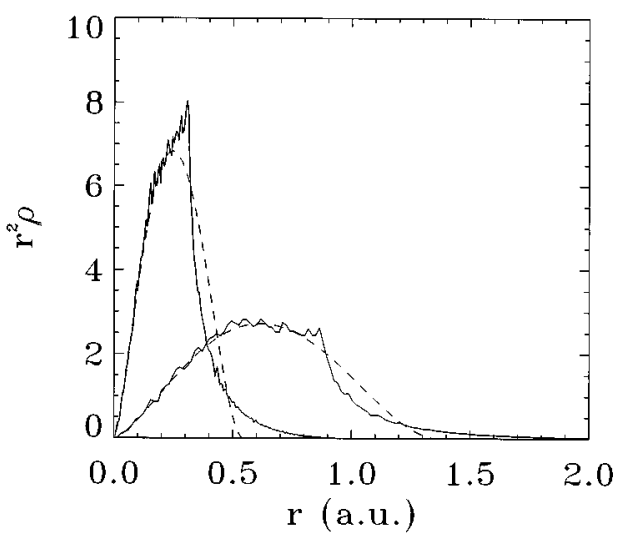

FIG. 4. Comparison of the electron density in STF theory (dashed curve) and the spherically averaged density in DM theory (solid curve) for $B=10^{11} \mathrm{G}$ (right) and $B=10^{12} \mathrm{G}$ (left).

of $\lambda_{c}$ is difficult and the values quoted should be regarded as lower bounds at the respective field strengths.

\section{COMPARISON WITH OTHER THEORIES}

As discussed in Sec. II, DM theory simplifies in the two limits, $\eta \rightarrow 0$ and $\eta \rightarrow \infty$, which are, respectively, described by the STF and HS theories. In order to study the rate of this convergence we have compared the ground-state energies at $\lambda=1$ in these three models in Table IV and plotted them in Fig. 3. It is remarkable how closely the STF ground-state energy approximates the DM energy even at fields as strong as $10^{15} \mathrm{G}$, while the electronic densities in DM theory deviate appreciably from the spherical shape of STF theory already at $10^{12} \mathrm{G}$ as seen in Fig. 1. Thus in this case at least, the energy calculations are much less sensitive to the details of the model than density calculations. There is, however, another way of comparing the densities in STF and DM theories. In Fig. 4 we have plotted together the STF density and the spherically averaged DM density, and one sees that they are quite close for the bulk of the electrons, up to fields of the order $10^{12} \mathrm{G}$. (The fine structure in the DM curve is partly a numerical artifact.)

It is apparent from Fig. 3 that the DM energy values approach the HS values as the field goes up, but the convergence is very slow and the asymptotic regime has not yet been reached at the strongest fields considered for the DM computations. On the other hand, it is interesting how well the HS density (23) fits the DM density integrated over the cross section of the atom as shown in Fig. 5.

Finally, in Tables V and VI we compare the ground-state energy $E$ computed in the DM and STF theories with values obtained by some other methods in the literature. The comparison is made for iron at $10^{12} \mathrm{G}$, since this case has been

TABLE V. Ground-state energy (in $\mathrm{keV}$ ) of iron atoms at $B=10^{12} \mathrm{G}$ according to DM theory, HF theory [17], DF computations, denoted $\mathrm{DF}^{a}$ [15] and $\mathrm{DF}^{b}$ [16], RV computations [23], TFD theory [12], and STF theory [8].

\begin{tabular}{lccccccc}
\hline \hline & $\mathrm{DM}$ & $\mathrm{HF}$ & $\mathrm{DF}^{a}$ & $\mathrm{DF}^{b}$ & $\mathrm{RV}$ & $\mathrm{TFD}$ & $\mathrm{STF}$ \\
$E$ & -54.38 & -55.10 & -56.10 & -58.3 & -53.13 & -56.21 & -54.07 \\
\hline \hline
\end{tabular}


TABLE VI. The composition of the ground-state energy $E$ (in $\mathrm{keV}$ ) of iron atoms at $B=10^{12} \mathrm{G}$ in STF theory, HF theory, and DM theory. $K$ is the kinetic energy, $A$ the attractive potential energy due to the nucleus, $R$ the energy of Coulomb repulsion, and $E_{\mathrm{ex}}$ the exchange energy.

\begin{tabular}{lclllc}
\hline \hline & $E$ & \multicolumn{1}{c}{$K$} & \multicolumn{1}{c}{$A$} & \multicolumn{1}{c}{$R$} & $E_{\mathrm{ex}}$ \\
\hline STF & -54.07 & 10.81 & -97.33 & 32.44 & 0 \\
HF & -55.10 & 10.6 & -95.4 & 32.7 & -3.06 \\
DM & -54.38 & 10.43 & -96.90 & 32.09 & 0 \\
\hline \hline
\end{tabular}

considered in a number of sources. In Table $\mathrm{V}$ the value for DM theory is compared with Hartree-Fock [18], density functional, [16,17] restricted variational (RV) [24], ThomasFermi-Dirac (TFD) [13], and STF calculations [9]. In Table $\mathrm{V}$ the splitting of the ground-state energy into its various parts (kinetic, attractive, repulsive, exchange) is compared for DM, STF, and HF calculations.

\section{CONCLUSIONS}

We have carried out a numerical study of the density matrix model that describes exactly the quantum mechanical ground state of atoms in a homogeneous magnetic field in the asymptotic limit when the nuclear charge $Z$, the electron number $N$, and the magnetic field $B$ tend to $\infty$ with $N / Z$ fixed and $B / Z^{4 / 3} \rightarrow \infty$. The calculations demonstrate the following features of heavy atoms in high magnetic fields as the field strength increases: A transition from an approximately spherical shape to a highly elongated shape, accompanied by a decrease in ground-state energy and increasing ability to bind excess electrons. We have also compared the DM model with the semiclassical Thomas-Fermi theory and the
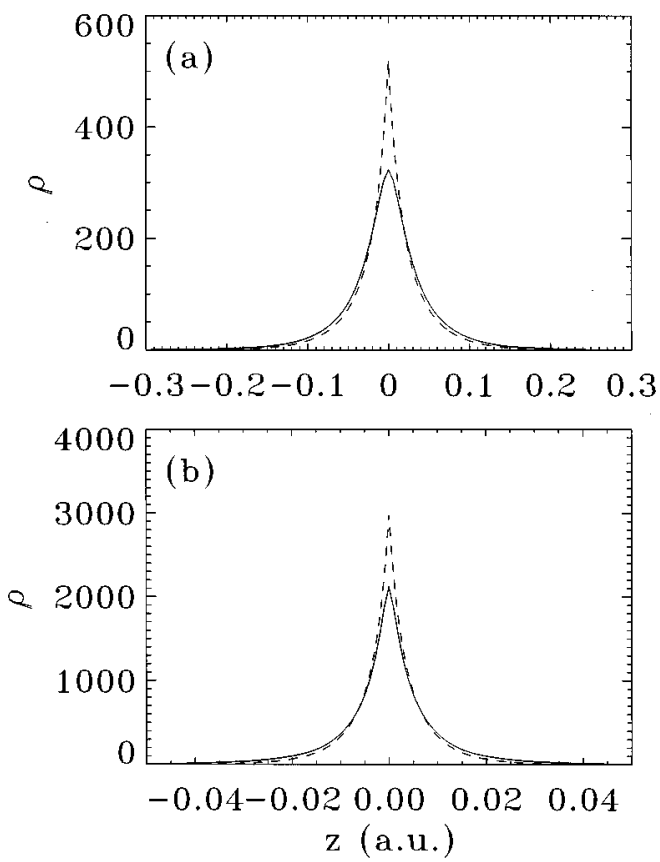

FIG. 5. Comparison of the electron density in HS theory (dashed curve) and the integral over $\mathbf{r}_{\perp}$ of the density in DM theory (solid curve) for $B=10^{13} \mathrm{G}$ (a) and $B=10^{18} \mathrm{G}(\mathrm{b})$. one-dimensional density functional theory that describe, respectively, its low and high field limits. When $\left(B / Z^{3}\right)^{1 / 5}$ is of order unity these simpler theories are numerically and conceptually wrong and the full DM theory should be used. The quantitative agreement of DM theory with Hartree-Fock calculations is quite good in strong fields; for iron at $B=10^{12} \mathrm{G}$ the difference in binding energies is less than $2 \%$. The DM theory, however, is much simpler computationally than HF theory for large atoms and appears suitable as a starting point for more refined approximations and for the study of molecular binding in strong fields.

\section{ACKNOWLEDGMENTS}

This work was supported by the Icelandic Science Foundation, the Foundation for Graduate Studies, and the Research Fund of the University of Iceland. We thank Sven Th. Sigurdsson and Chris Pethick for helpful advice and valuable comments.

\section{APPENDIX}

We give here a proof of Eqs. (21) and (22). It consists essentially of an improvement of the estimates in Proposition 3.3 in [4].

With $L(\eta)$ defined by (20) we define for any density $\rho$ a rescaled density $\rho_{\eta}$ by

$$
\rho\left(\mathbf{r}_{\perp}, z\right)=Z^{4} \eta L(\eta) \rho_{\eta}\left(Z \eta^{1 / 2} \mathbf{r}_{\perp}, Z L(\eta) z\right) .
$$

We can then write the SS functional (15) as

$$
\mathcal{E}^{\mathrm{SS}}[\rho]=Z^{3} L(\eta)^{2} \mathcal{E}_{\eta}^{\mathrm{SS}}\left[\rho_{\eta}\right]
$$

where $\mathcal{E}_{\eta}^{\text {SS }}$ is defined by

$$
\begin{aligned}
\mathcal{E}_{\eta}^{\mathrm{SS}}\left[\rho_{\eta}\right]= & \int\left(\frac{\partial \sqrt{\rho_{\eta}}}{\partial z}\right)^{2} d^{3} \mathbf{r}-\int \rho_{\eta}(\mathbf{r}) V_{\eta}(\mathbf{r}) d^{3} \mathbf{r} \\
& +\frac{1}{2} \iint \rho_{\eta}(\mathbf{r}) V_{\eta}\left(\mathbf{r}-\mathbf{r}^{\prime}\right) \rho_{\eta}\left(\mathbf{r}^{\prime}\right) d^{3} \mathbf{r} d^{3} \mathbf{r}^{\prime},
\end{aligned}
$$

with the rescaled Coulomb potential

$$
V_{\eta}(\mathbf{r})=L(\eta)^{-1}\left[z^{2}+\eta^{-1} L(\eta)^{2} \mathbf{r}_{\perp}^{2}\right]^{-1 / 2} .
$$

The functional $\mathcal{E}_{\eta}^{\mathrm{SS}}$ has a ground-state energy

$$
\begin{gathered}
E_{\eta}^{\mathrm{SS}}(\lambda)=\inf \left\{\mathcal{E}_{\eta}^{\mathrm{SS}}\left[\rho_{\eta}\right]: \rho_{\eta} \in \mathcal{C}^{\mathrm{SS}}, \quad \int \rho_{\eta} \leqslant \lambda,\right. \\
\left.\qquad \rho_{\eta}\left(\mathbf{r}_{\perp}, z\right) d z \leqslant 1\right\}
\end{gathered}
$$

and a corresponding minimizing density denoted by $\rho_{\eta}^{\mathrm{SS}}$ It is related to $\rho^{\mathrm{SS}}$ by the scaling (A1). The energy $E_{\eta}^{\mathrm{SS}}$ is related to $E^{\mathrm{SS}}$ by the scaling (A2),

$$
E^{\mathrm{SS}}(N, Z, B)=Z^{3} L(\eta)^{2} E_{\eta}^{\mathrm{SS}}(\lambda) .
$$

The improvement of Proposition 3.3 in [4] is stated in the following lemma. 
Lemma A.1. For any choice of $\lambda$ and $T$ there is a constant $C(\lambda, T)$ such that

$$
\left|\int \rho\left(\mathbf{r}_{\perp}, 0\right) d^{2} \mathbf{r}_{\perp}-\int V_{\eta} \rho\right| \leqslant C(\lambda, T) L(\eta)^{-1}
$$

holds, provided $\rho \geqslant 0$ satisfies $\int \rho \leqslant \lambda, \quad \rho\left(\mathbf{r}_{\perp}, z\right)=0$ for $\left|\mathbf{r}_{\perp}\right|>\sqrt{2}$, and

$$
T[\rho]=\int\left(\frac{\partial \sqrt{\rho\left(\mathbf{r}_{\perp}, z\right)}}{\partial z}\right)^{2} d^{3} \mathbf{r} \leqslant T .
$$

We can choose $C(\lambda, T)=\lambda+8 \sqrt{2} \lambda^{1 / 4} T^{3 / 4}+8 \sqrt{\pi} T^{1 / 2}$ $\times \ln (\sqrt{2}+\sqrt{6})$.

Proof : Following the proof of Proposition 3.3 [4] we write the difference on the left side of (A7) as $A_{1}+A_{2}+A_{3}$ with

$$
\begin{gathered}
A_{1}=-\int_{\left|\mathbf{r}_{\perp}\right| \geqslant 1} V_{\eta}(\mathbf{r}) \rho(\mathbf{r}) d^{3} \mathbf{r} \\
A_{2}=\int_{\left|\mathbf{r}_{\perp}\right| \leqslant 1} V_{\eta}(\mathbf{r})\left[\rho\left(\mathbf{r}_{\perp}, 0\right)-\rho(\mathbf{r})\right] d^{3} \mathbf{r},
\end{gathered}
$$

and

$$
A_{3}=\int\left[1-\int_{\left|\mathbf{r}_{\perp}\right| \leqslant 1} V_{\eta}(\mathbf{r}) d z\right] \rho\left(\mathbf{r}_{\perp}, 0\right) d^{2} \mathbf{r}_{\perp} .
$$

With the same arguments as in [4] we obtain

$$
\begin{gathered}
\left|A_{1}\right| \leqslant \frac{\lambda}{L(\eta)}, \\
\left|A_{2}\right| \leqslant \frac{8 \sqrt{2}}{L(\eta)} \lambda^{1 / 4} T^{3 / 4},
\end{gathered}
$$

and $^{6}$

$$
\begin{aligned}
\left|A_{3}\right|= & \left|\int\left[\frac{2}{L(\eta)} \sinh ^{-1}\left\{\eta^{1 / 2} /\left[L(\eta)\left|\mathbf{r}_{\perp}\right|\right]\right\}-1\right] \rho\left(\mathbf{r}_{\perp}, 0\right) d^{2} \mathbf{r}_{\perp}\right| \\
\leqslant & 2 T^{1 / 2} \sqrt{2 \pi}\left\{\int _ { 0 } ^ { \sqrt { 2 } } \left[\frac{2}{L(\eta)}\right.\right. \\
& \left.\left.\times \sinh ^{-1}\left\{\eta^{1 / 2} /[L(\eta) r]\right\}-1\right]^{2} r d r\right\}^{1 / 2} .
\end{aligned}
$$

Now we deviate from [4]. Estimating the integral in (A13) we obtain

$$
\begin{aligned}
\left|A_{3}\right| \leqslant & 2 \sqrt{2 \pi} T^{1 / 2} 2^{1 / 4} \sup _{0 \leqslant r \leqslant \sqrt{2}} \mid\left[\frac{2}{L(\eta)}\right. \\
& \left.\times \sinh ^{-1}\left\{\eta^{1 / 2} /[L(\eta) r]\right\}-1\right] r^{1 / 2} \mid \\
\leqslant & 8 \sqrt{\pi} T^{1 / 2} \ln (\sqrt{2}+\sqrt{6}) / L(\eta) .
\end{aligned}
$$

The last inequality comes from the following. If $\alpha=\sqrt{\eta} / L(\eta)$, then

\footnotetext{
${ }^{6}$ In [4], p. 541 there is a power of $1 / 2$ missing on $T$ in the estimate for $A_{3}$.
}

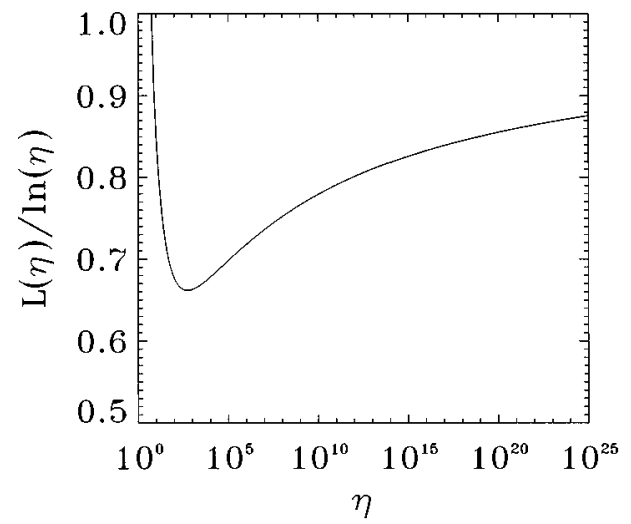

FIG. 6. The ratio $L(\eta) / \ln \eta$.

$$
2 \sinh ^{-1}(\alpha / \sqrt{2})-L(\eta)=0
$$

by the definition (20) of $L(\eta)$. Using (A15) we get

$$
\begin{aligned}
\sup _{0 \leqslant r \leqslant \sqrt{2}}\left|\left[2 \sinh ^{-1}(\alpha / r)-L(\eta)\right] r^{1 / 2}\right| \\
=\sup _{0 \leqslant r \leqslant \sqrt{2}} \mid\left[2 \sinh ^{-1}(\alpha / r)-2 \sinh ^{-1}(\alpha / \sqrt{2})\right. \\
\left.+2 \sinh ^{-1}(\alpha / \sqrt{2})-L(\eta)\right] r^{1 / 2} \mid \\
=\sup _{0 \leqslant r \leqslant \sqrt{2}}\left|\left[2 \sinh ^{-1}(\alpha / r)-2 \sinh ^{-1}(\alpha / \sqrt{2})\right] r^{1 / 2}\right| \\
\left.=2 \sup _{0 \leqslant r \leqslant \sqrt{2}} \mid\left[\ln (\sqrt{2} / r)+\ln \mid \frac{\alpha+\sqrt{\alpha^{2}+r^{2}}}{\alpha+\sqrt{\alpha^{2}+2}}\right)\right] r^{1 / 2} \mid \\
\leqslant 2 \sup _{0 \leqslant r \leqslant \sqrt{2}}\left|\ln (\sqrt{2} / r) r^{1 / 2}\right| \\
+2 \sup _{0 \leqslant r \leqslant \sqrt{2}}\left|\ln \left(\frac{\alpha+\sqrt{\alpha^{2}+r^{2}}}{\alpha+\sqrt{\alpha^{2}+2}}\right) r^{1 / 2}\right| \\
\leqslant 2^{5 / 4} \ln 2+2^{5 / 4} \ln [(1+\sqrt{3}) / \sqrt{2}]=2^{5 / 4} \ln (\sqrt{2}+\sqrt{6}) .
\end{aligned}
$$

This concludes the proof.

In analogy with Proposition 3.4 in [4] the following lemma is a corollary of Lemma A.1.

Lemma A.2. For $\rho$ as in Lemma A.1 there is a constant $C^{\prime}(\lambda, T)$ such that

$$
\begin{aligned}
& \left|\iint \rho(\mathbf{r}) V_{\eta}\left(\mathbf{r}-\mathbf{r}^{\prime}\right) \rho\left(\mathbf{r}^{\prime}\right) d^{3} \mathbf{r} d^{3} \mathbf{r}^{\prime}-\int \bar{\rho}(z)^{2} d z\right| \\
& \quad \leqslant C^{\prime}(\lambda, T) L(\eta)^{-1},
\end{aligned}
$$

where $\bar{\rho}(z)=\int \rho\left(\mathbf{r}_{\perp}, z\right) d^{2} \mathbf{r}_{\perp}$.

The proof of (21) and (22) is now identical to the proof of Theorem 3.4 in [4] with Lemmas A.1 and A.2 in place of Propositions 3.3 and 3.4.

In order to illustrate the difference between the function $L(\eta)$ and the approximation $L(\eta) \approx \ln \eta$ used in [4], the ratio $L(\eta) / \ln \eta$ is plotted in Fig. 6. 
[1] G. Chanmugam, Annu. Rev. Astron. Astrophys. 30, 143 (1992).

[2] P. Mészáros, High-Energy Radiation from Magnetized Neutron Stars (University of Chicago Press, Chicago, 1992).

[3] H. Ruder, G. Wunner, H. Herold, and F. Geyer, Atoms in Strong Magnetic Fields (Springer, Berlin, 1994).

[4] E.H. Lieb, J.P. Solovej, and J. Yngvason, Commun. Pure Appl. Math. 47, 513 (1994).

[5] E.H. Lieb, J.P. Solovej, and J. Yngvason, Commun. Math. Phys. 161, 77 (1994).

[6] E.H. Lieb, J.P. Solovej, and J. Yngvason, Phys. Rev. Lett. 69, 749 (1992).

[7] B.B. Kadomtsev, Zh. Éksp. Teor. Fiz. 58, 1765 (1970) [Sov. Phys. JETP 31, 945 (1970)].

[8] R.O. Mueller, A.R.P. Rau, and L. Spruch, Phys. Rev. Lett. 26, 1136 (1971).

[9] B. Banerjee, D.H. Constantinescu, and P. Rehák, Phys. Rev. D 10, 2384 (1974).

[10] I. Fushiki, E.H. Gudmundsson, and C.J. Pethick, Astrophys. J. 342, 958 (1989).

[11] A.M. Abrahams and S.L. Shapiro, Astrophys. J. 374, 652 (1991).

[12] Ö.E. Rögnvaldsson, I. Fushiki, C.J. Pethick, E.H. Gudmunds- son, and J. Yngvason, Astrophys. J. 216, 276 (1993).

[13] J.E. Skjervold and E. Östgaard, Phys. Scr. 29, 543 (1984).

[14] B.B. Kadomtsev and V.S. Kudryavtsev, Zh. Éksp. Teor. Fiz. 13, 61 (1971) [Sov. Phys. JETP 13, 42 (1971)].

[15] B.B. Kadomtsev and V.S. Kudryavtsev, Pis'ma Zh. Éksp. Teor. Fiz. 13, 15 (1971) [JETP Lett. 13, 9 (1971)].

[16] P. B. Jones, Mon. Not. R. Astron. Soc. 216, 503 (1985).

[17] D. Kössl, R.G. Wolff, E. Müller, and W. Hillebrandt, Astron. Astrophys. 205, 347 (1988).

[18] D. Neuhauser, S.E. Koonin, and K. Langanke, Phys. Rev. A 342, 4163 (1987).

[19] B.G. Englert, Semiclassical Theory of Atoms, Lecture Notes in Physics Vol. 300 (Springer, Berlin, 1988).

[20] E.H. Lieb, Rev. Mod. Phys. 53, 603 (1981); Rev. Mod. Phys. 54, 311(E) (1982).

[21] J.E. Avron, I. Herbst, and B. Simon, Commun. Math. Phys. 79, 529 (1981).

[22] L.R. Ram-Moham, S. Saigal, D. Dossa, and J. Shertzer, Comput. Phys. 4, 50 (1990).

[23] W.H. Press, S.A. Teukolsky, W.T. Vetterling, and B.P. Flannery, Numerical Recipes in $C$ (Cambridge University Press, New York, 1992).

[24] E. Müller, Astron. Astrophys. 130, 415 (1984). 